\title{
CÁLCULOS DE EQUILÍBRIO EM ACIARIA ATRAVÉS DA TERMODINÂMICA COMPUTACIONAL
}

André Luiz Vasconcellos da Costa e Silva 1

\section{Resumo}

Embora durante muito tempo o desenvolvimento dos processos de aciaria tenha se dado de forma bastante empírica, o projeto e controle destes processos dependem do conhecimento do estado de equilíbrio termodinâmico dos sistemas em questão. A complexidade destes problemas de equilíbrio, entretanto, limita, em muitos casos, a possibilidade de soluções por métodos simples. A termodinâmica computacional viabiliza a solução destes problemas com um número limitado de simplificações, uma mudança significativa em relação aos métodos até então aplicados. No presente trabalho, através de exemplos de equilíbrios relevantes, é demonstrado como a aplicação da termodinâmica computacional permite determinar 0 estado de equilíbrio de sistemas importantes para o aciarista. Casos em que a termodinâmica indica apenas tendências, tais como a avaliação da segregação de solidificação, são também discutidos. Conclui-se que o emprego da termodinâmica computacional viabiliza a avaliação de forma relativamente simples e direta de vários equilíbrios importantes em aciaria. Estas avaliações podem ser decisivas para reduzir o tempo e os gastos no desenvolvimento de novas rotas de elaboração e de novos aços.

Palavras-chave: Termodinâmica computacional; Inclusão não-metálica; Segregação.

\section{EQUILIBRIUM CALCULATIONS IN STEELMAKING USING COMPUTATIONAL THERMODYNAMICS}

\begin{abstract}
Although for a long period of time steelmaking process development has been mostly empirical, it is now well established that process design and control are closely dependent on the knowledge of the equilibrium conditions. Equilibrium calculations for the multi-phase, multi-component systems relevant to steelmaking can only be handled using computational aides, except for the simplest cases. In the present work the possibility of using computational thermodynamics to solve melt shop relevant problems is demonstrated through selected examples. Situations in which thermodynamics can only indicate the directions and trends are also discussed. It is concluded that the use of computational thermodynamics makes possible the relatively simple and direct evaluation of several important equilibria in the steel melt shop. These evaluations can be powerful and decisive tools in reducing development cost and time when designing or improving processes or steels.
\end{abstract}

Key words: computational thermodynamics, non-metallic inclusions, segregation.

\section{INTRODUÇÃO}

Durante um longo período do século passado os processos de aciaria foram desenvolvidos de forma basicamente empírica. $\mathrm{Na}$ década de 1940 iniciou-se o desenvolvimento significativo de metodologias para o tratamento de problemas de equilíbrio termodinâmico em ligas metálicas líquidas e, no final da década de 1960 a aplicação sistemática de modelos físicos e matemáticos, aliada ao desenvolvimento da área computacional, começou a viabilizar a compreensão dos principais fenômenos relevantes para a eficiência dos processos de aciaria.

Parte da complexidade associada a este desenvolvimento certamente se deveu às elevadas temperaturas de processo, que dificultam desde a simples observação do processo até a realização de medidas fundamentais ao acompanhamento e compreensão do processo. Adicionalmente, boa parte dos processos mais eficientes opera em condições distantes daquelas de equilíbrio termo- dinâmico e, embora 0 atual estado do conhecimento da cinética destes processos permita compreender este desvio do equilíbrio, muitas explicações preliminares foram baseadas em premissas que contrariavam a termodinâmica do processo. Hoje é bem estabelecido que o conhecimento do estado de equilíbrio, "para onde 0 sistema vai" é fundamental para o modelamento e compreensão dos processos que se passam e são concluídos ainda fora de equilíbrio. Por outro lado, processos que se passam próximo ao equilíbrio, ou em que o equilíbrio termodinâmico é, praticamente, atingido, envolvem sistemas muito complexos, multi-componente e, em geral, duas ou mais fases, não sendo tratáveis pelos métodos simplificados desenvolvidos antes da década de 1970.

\footnotetext{
1 M embro da ABM, Engenheiro M etalúrgico, PhD, Diretor Técnico do IBQN, Professor da EEIM VR-UFF, Volta Redonda RJ, andre@ metal.eeimvr.uff.br
} 
Este cenário levou a duas conseqüências importantes: a) fomentou o desenvolvimento de modelos empíricos ou semi-empíricos para o controle e projeto de processos de aciaria, com as limitações associadas a este empirismo, e b) consolidou um relativo descrédito na capacidade da termodinâmica em auxiliar no desenvolvimento e compreensão dos processos que ocorrem na aciaria. A ênfase dada, no ensino da termodinâmica, aos métodos de cálculo baseados em enfoques simplificadores, ao invés da valorização dos conceitos fundamentais e da termodinâmica como ciência, contribuíram para esta segunda conseqüência. Mesmo depois do desenvolvimento de técnicas alternativas mais modernas, esta situação pouco se alterou. Assim, embora técnicas de cálculo termodinâmico automatizadas estejam estabelecidas e disponíveis desde, pelo menos, a década de 1980, sua introdução na aciaria vem sendo lenta. Embora as ferramentas de cálculo disponíveis não sejam, em sua maioria, muito "amigáveis" (user-friendly) 0 autor acredita que a maior barreira à sua implantação em mais larga escala na siderurgia e, em particular, na aciaria, esteja ligada a dificuldade do ensino da termodinâmica se adaptar à disponibilidade dos novos métodos e novas ferramentas.

N este trabalho são apresentadas e discutidas algumas aplicações da chamada "termodinâmica computacional" à solução de problemas de aciaria, destacando as vantagens e limitações desta técnica quando comparada às principais alternativas para a avaliação do estado de equilíbrio nos processos.

\section{EQUILÍBRIOS EM ACIARIA}

Os principais equilíbrios importantes em aciaria envolvem sistemas multi-fase tais como: metal-óxido (metal-escória, metal-refratário, metal-inclusões não-metálicas), metal líquidometal sólido, metal-gás. N estes sistemas, em vista da complexidade das ligas à base de ferro e dos processos de refino, todas as fases são multicomponentes, isto é, contém vários elementos químicos ou "espécies". Assim, além da necessidade de se conhecer a tendência à reação química entre os elementos ou "espécies" presentes, é necessário conhecer como estes elementos ou espécies se comportam quando se encontram em solução em uma fase multi-componente.

Em função das elevadas temperaturas e da pressão entre moderada e baixa reinante nos processos de aciaria, o tratamento das misturas de gases é bastante simples, podendo ser realizado como se fossem misturas de gases ideais.

\subsection{Soluções Metálicas - 0 Enfoque Clássico}

0 tratamento da termodinâmica das soluções metálicas apresenta uma maior complexidade, pois estas se desviam significativamente do comportamento ideal. Evidência clara deste desvio é o chamado "efeito térmico das ferroligas" conhecido por todo aciarista. A dissolução do silício no aço, por exemplo, produz um efeito muito diferente, sobre a temperatura do sistema, do que a dissolução do manganês. Assim, foi preciso desenvolver uma metodologia que viabilizasse 0 cálculo da "tendência à reação química" (ou atividade) dos elementos em solução nos aços.

U m método de tratamento matemático que minimizasse as operações matemáticas necessárias ao cálculo foi desenvolvido e consolidado na década de $1950 .(1,2) \mathrm{N}$ esta formulação, privilegiouse a tabulação de valores que dependessem linearmente da temperatura e da composição química, considerando as ferramentas computacionais da época: papel, lápis, régua de cálculo e tábua de logaritmos.

A variação de energia livre associada ao processo de solução de um elemento no ferro liquido era expressa através de um coeficiente de atividade da lei de Henry $\left(\gamma_{i}^{\mathrm{T}}\right)$ ou de uma "variação de energia livre de formação da solução a 1\%" $\left(\Delta G_{e}^{\mathrm{kN}}\right)$, relacionados através de:

$$
\Delta G_{i}^{1 N}=R T \ln \frac{\gamma_{i}^{0} M_{F_{e}}}{100 M_{i}}
$$

U ma evidência da busca pela praticidade dos cálculos está no fato que, até hoje, só se encontra valores tabelados para 0 efeito da temperatura sobre $\Delta G_{\sigma}^{\mathrm{m}}$, que é linear, enquanto $\gamma^{\circ}$ é tabelado para 1873K, evitando a expressão da variação exponencial com a temperatura, uma dificuldade de calculo na era "pré-calculadora eletrônica".

$A$ atividade de um elemento i, em solução no ferro, na presença de outros solutos j era então expressa pelo produto de dois termos: um termo que expressa 0 efeito da composição química do soluto e o outro, um coeficiente que reflete 0 efeito das interações entre os solutos. Este coeficiente, que depende dos teores dos demais solutos, era obtido através de uma expansão em série de Taylor do seu logaritmo decimal, visando, também, simplificar, tanto quanto possível, os cálculos.

$$
\begin{aligned}
& a_{i}=f_{i} \%_{i} \quad \log a_{i}-\log f_{i}+\log \%_{i} \\
& \log f_{i}=\sum_{i} e_{i}^{j} \xi_{j}+\sum_{i} r_{i}^{j}\left(\xi_{j}\right)^{2}+\ldots . \cdot
\end{aligned}
$$

É evidente que, ainda assim, os cálculos resultantes não eram nada convidativos. Se, por exemplo, as interações de primeira ordem tivessem de ser consideradas, não era possível obter uma solução analítica, e o método de tentativa e erro era 0 único caminho possível. Naturalmente, a termodinâmica das soluções metálicas liquidas, mesmo simplificada por Wagner, Chipman, Elliott e outros, não se tornou um assunto muito popular nas aciarias. 


\subsection{Problema das Misturas de Óxidos}

Nos casos em que a presença de óxidos deve ser considerada, a situação se torna ainda mais complexa. A dificuldade em conhecer a estrutura das escórias e das misturas de óxidos, limita a possibilidade de prever a tendência a reagir dos óxidos presentes em escórias, refratários e inclusões não-metálicas, exceto nas situações mais simples (saturação e compostos de composição definida). Embora modelos termodinâmicos para prever 0 comportamento dos óxidos nestas misturas tenham sido formulados já na década de 1960,(3) nenhum destes modelos é passivel de tratamento suficientemente simplificado para uso com as ferramentas "pré-calculadora". Estabeleceu-se como alternativa a consulta a diagramas de fases ternários e, eventualmente a formulas empíricas que "ocultavam" o modelo físico-químico que descreveria o comportamento da escória (p.ex. modelo de Healy para desfosforação).(4)

O cenário "pré-calculadora" é, portanto, caracterizado pela necessidade de uso de dois ou três modelos termodinâmicos completamente diferentes para a previsão do comportamento dos solutos (dependendo da fase: gás, metal líquido ou óxidos-escória, refratário, inclusões), consulta a tabelas de dados e coeficientes e, freqüentemente, consulta a diversos diagramas. 0 esforço envolvido na compreensão do problema, no aprendizado dos métodos de solução e na localização e consulta dos diagramas e tabelas de dados raramente era justificado pelos resultados obtidos pelo aciarista "típico".

\subsection{A "Termodinâmica Computacional”}

A percepção de que a determinação do estado de equilíbrio em sistemas com diversos componentes e várias fases, com a precisão necessária para ser útil ao aciarista, não é prática com 0 emprego dos métodos descritos acima, aliada a disponibilidade de computadores e calculadoras a partir da década de 1970, levou ao desenvolvimento da chamada "termodinâmica computacional". 0 conceito termodinâmico fundamental envolvido é a percepção de que "em um sistema multi-componente, multi-fásico, a temperatura e pressão constantes, as fases presentes, suas composições e quantidades se ajustarão de forma a atingir um mínimo para 0 valor de uma função termodinâmica do sistema, a sua energia livre de Gibbs".

Assim, existem dois problemas importantes na termodinâmica computacional:

Como descrever a energia livre de Gibbs de cada uma das fases, em função da temperatura, pressão e composição química e

Como calcular a combinação de fases, suas composições e quantidades que resultarão em um mínimo da energia livre de Gibbs do sistema.

O segundo problema é, fundamentalmente, um problema matemático e não de termodinâmica. Para o engenheiro metalúrgico ou aciarista compreendê-lo é tão relevante como compreender os algoritmos de programação linear que conduzem a composição de carga de custo mínimo para uma aciaria elétrica.

0 primeiro problema tem duas vertentes: Em primeiro lugar, é preciso formular modelos matemáticos que descrevam de forma satisfatória o comportamento das soluções reais. Presen- temente, para cada tipo de fase envolvida nos processos de aciaria (metal liquido, metal sólido, escória, inclusões, refratários e gás) há, pelo menos, dois modelos alternativos capazes de fornecer resultados satisfatórios. Em segundo lugar, é preciso ajustar os coeficientes dos modelos às medidas experimentais. A termodinâmica ainda é uma ciência cuja capacidade de fazer previsões é limitada pela existência de dados experimentais. Uma vez ajustados os coeficientes de determinado modelo à determinada fase, armazena-se esta informação em "bancos de dados" termodinâmicos. Como as funções termodinâmicas de cada fase são relacionadas entre si,(5) basta escolher uma das funções para a constituição do banco de dados. A maior parte dos bancos de dados comercialmente disponíveis armazena descrições polinomiais da energia livre de Gibbs das fases, em função da composição química e da temperatura (e, no caso dos gases, da pressão).

Para o aciarista (ou metalurgista) moderno, é suficiente conhecer: (a) a estrutura da termodinâmica computacional, (b) 0 que significa "o mínimo da energia livre de Gibbs" e (c) como esta condição de equilíbrio se reflete no equilíbrio entre fases. Ao usar um programa de termodinâmica computacional, não é importante, para o usuário, conhecer em detalhe qual o modelo empregado para descrever cada fase, desde que este tenha sido adequadamente selecionado por quem desenvolveu 0 programa. Da mesma forma, não é necessário buscar dados em tabelas ou gráficos, pois estas informações devem ter sido as fontes usadas no ajuste dos coeficientes durante 0 desenvolvimento do banco de dados. É necessário, entretanto, conhecer as limitações de uso estabelecidas por quem desenvolveu 0 banco de dados (faixas de composição química, de temperatura, etc.). Extrapolações alem dos limites recomendados podem levar a resultados nãoconfiáveis.

\section{EXEMPLOS DE APLICAÇÃO}

\subsection{Formulação de Problemas Simples}

0 primeiro ponto importante na aplicação da termodinâmica à aciaria é, naturalmente, formular da melhor maneira possível 0 problema em questão. Como na termodinâmica computacional não existem as limitações usuais (associadas ao método de cálculo), a formulação pode ser o mais objetiva possível, como indicado nos dois exemplos abaixo. 
É essencial compreender que o cálculo de qualquer equilíbrio implica na solução de um sistema de equações simultâneas. A matemática indica que há uma relação entre o número de incógnitas e o número de equações para que um sistema de equações tenha solução: havendo incógnitas demais, o sistema não pode ser resolvido de forma única e, havendo equações demais, o problema só pode ser resolvido se as equações forem dependentes. Em termodinâmica, a relação entre o número de equações a resolver e o número de variáveis é expresso através da regra das fases de Gibbs:

$$
P+F=C+2 \text { ou } F=C-P+2
$$

U sualmente, em termodinâmica computacional, interpreta-se esta regra como estabelecendo que: "Se não definimos a priori que determinada fase deve estar presente, precisamos definir $\mathrm{C}+2$ variáveis (onde $\mathrm{C}$ é 0 número de constituintes ou elementos) para que o sistema de equações tenha solução possível e única".

\subsubsection{Desoxidação por alumínio}

Supondo que a desoxidação por alumínio de um aço se passe até atingir o equilíbrio, a quantidade de alumínio a adicionar, no vazamento, é definida pelo teor de oxigênio inicial do aço e o teor de oxigênio desejado, em solução no aço, ao fim da desoxidação. 0 problema pode ser formulado, então, como indicado na Tabela 1.

0 resultado indica que o óxido formado será alumina e que a quantidade teórica de alumínio a adicionar para esta desoxidação é de $0,875 \mathrm{~kg} / \mathrm{t}$ de aço. $\mathrm{N}$ aturalmente, neste exemplo, o rendimento do alumínio e a eventual presença de escória não foram considerados, para facilitar a compreensão da sua formulação. A solução deste problema pelos métodos clássicos envolveria duas etapas: 0 cálculo do teor de alumínio em solução, ao final da desoxidação, em equilíbrio com alumina e o cálculo do alumínio consumido (estequiometricamente) na formação da alumina até atingir tal equilíbrio. Além disto, seria necessário assumir, a priori, qual o óxido que se forma, (a alumina, neste exemplo) ou repetir o cálculo para todos os óxidos possíveis no sistema. A formulação do problema, através da termodinâmica computacional é mais direta, não envolvendo duas etapas de cálculo, e não requerendo a escolha, a priori, do óxido a ser considerado como o produto da desoxidação.

\subsubsection{Efeito térmico da adição de Fe-Si ao aço}

A adição de ferroligas ao aço causa variação de sua temperatura. Supondo que se adicione $0,3 \%$ de silício a um aço, sob a forma de ferroliga $\mathrm{Fe}-\mathrm{Si} 70 \%$, qual seria a temperatura final do aço?. Este problema é resolvido, normalmente, através de um balanço térmico, em que se assume a conservação de energia. Como a adição se passa a pressão constante, a formulação do problema de conservação de energia é expressa pela condição de que a entalpia do sistema é constante.

A solução clássica deste balanço térmico requereria, no mínimo, informações sobre: a) a entalpia de formação (ou dissociação) dos compostos Fe-Si presentes na ferroliga, b) a variação da entalpia na mistura de Fe e Si nos estados sólido e líquido, c) informações sobre os calores específicos (ou entalpias) do ferro puro, do silício puro e das misturas $\mathrm{Fe}$-Si na faixa de temperatura contemplada, e d) as variações de entalpia em transformações de fases (fusão do ferro e do silício, por exemplo). Se os dados disponíveis estivessem sob a forma de calores específicos, a solução clássica do balanço térmico envolveria a determinação do limite de integração de uma integral relativamente complexa. A simplicidade da formulação do problema e de sua solução através da termodinâmica computacional é evidente, e destaca o conceito fundamental envolvido, a conservação da energia, expressa pela condição de igualdade entre a entalpia inicial e a entalpia final.

Tabela 1. Calculo da adição teórica de alumínio para a desoxidação de um aço.

\begin{tabular}{ll}
\hline Condições definidas para o Cálculo & Resultado do Cálculo \\
\hline Sistema composto por Fe-Al- $\mathrm{O}, \mathrm{C}=3$. & a)Fases Presentes: \\
Condições $(\mathrm{F}=5):$ & $998,7 \mathrm{~kg}$ de aço líquido contendo \\
Tamanho do sistema $=10^{6} \mathrm{~g}(1 \mathrm{t}$ de aço $)$ & $0=6 \mathrm{ppm} \% \mathrm{Al}=0,02 \%$ \\
$\% 0$ total $=600 \mathrm{ppm}$ & $1,3 \mathrm{~kg}$ de Alumina $\left(\mathrm{Al}_{2} \mathrm{O}_{3}\right)$ \\
$\% 0$ no aço $=6 \mathrm{ppm}$ & b) Q uantidade total de alumínio no \\
$\mathrm{T}=1873 \mathrm{~K}\left(1600^{\circ} \mathrm{C}\right) \mathrm{P}=10^{5} \mathrm{~Pa}(1 \mathrm{~atm})$ & sistema: $875 \mathrm{~g}$ \\
\hline
\end{tabular}

Tabela 2. Calculo do efeito térmico de uma adição de Fe-Si

\begin{tabular}{|c|c|}
\hline $\begin{array}{l}\text { Condições definidas para o Cálculo } \\
\text { Sistema composto por Fe-Si, C = } 2 \text {. }\end{array}$ & Resultado do Cálculo \\
\hline $\begin{array}{l}\text { a) Primeiro cálculo: } \\
\text { Aço sem silício. Condições }(\mathrm{F}=4) \text { : } \\
\text { Tamanho do sistema }=10^{6} \mathrm{~g}(1 \mathrm{t} \text { de Fe }) \\
\% \text { Si no aço }=0 \\
\mathrm{~T}=1873 \mathrm{~K}(1600 \mathrm{CC}) \mathrm{P}=10^{5} \mathrm{~Pa}(1 \mathrm{~atm})\end{array}$ & $\begin{array}{l}\text { a) Fases Presentes: } \\
1000 \mathrm{~kg} \text { de aço líquido } \\
\text { Entalpia }=1,34 \times 10^{9} \mathrm{j}\end{array}$ \\
\hline $\begin{array}{l}\text { b) Segundo cálculo } \\
\text { Liga Fe-Si (para a adição de } 3 \mathrm{~kg} \text { de Si) } \\
\text { Condições }(\mathrm{F}=4) \text { : } \\
\text { Tamanho do sistema= } 4286 \mathrm{~g} \text { de Fe-Si } \\
\% \text { Si na liga }=70 \\
\mathrm{~T}=298 \mathrm{~K}\left(25^{\circ} \mathrm{C}\right) \quad \mathrm{P}=10^{5} \mathrm{~Pa}(1 \mathrm{~atm})\end{array}$ & $\begin{array}{l}\text { b) Fases presentes: } \\
\text { Si metálico e FeSi } \\
\text { Entalpia= }-1,46 \times 10^{6} \mathrm{~J}\end{array}$ \\
\hline $\begin{array}{l}\text { c) Terceiro cálculo } \\
\text { Aço com Si. Condições }(F=4) \text { : } \\
\text { Tamanho do sistema }=10^{6}+4286 \mathrm{~g} \\
\% \text { Si no aço }=0,3 \mathrm{P}=10^{5} \mathrm{~Pa}(1 \mathrm{~atm}) \\
\text { Entalpia total }=1,34 \times 10^{9} \mathrm{~J}+\left(-1,46 \times 10^{6} \mathrm{~J}\right)\end{array}$ & $\begin{array}{l}\text { c) Fases presentes: } \\
\tilde{=} 1,0043 \times 106 \mathrm{~g} \text { de aço líquido } \\
\text { contendo } \% \mathrm{Si}=0,3 \\
\mathrm{~T}=1870.31 \mathrm{~K}(1597.16 \mathrm{C})\end{array}$ \\
\hline
\end{tabular}




\subsection{Problemas Aplicados}

\subsubsection{Saturação da escória do conversor em Mgo}

Conhecer a solubilidade do M go em escórias de conversor é importante para o controle da agressividade aos refratários. Varias correlações empíricas já foram apresentadas na literatura e revistas por Chrisóstomo e colaboradores(6). A complexidade experimental limita o número de fatores que podem ser tratados nestas formulas empíricas. Chrisóstomo e colaboradores calcularam, através da termodinâmica computacional, a temperatura liquidus de escórias contendo $\mathrm{CaO}, \mathrm{FeO}, \mathrm{Fe}_{2} \mathrm{O}_{3}, \mathrm{Al}_{2} \mathrm{O}_{3}, \mathrm{SiO}_{2}$, $\mathrm{MgO}$ e $\mathrm{MnO}$. Para fins práticos, foi realizada uma regressão multivariada da superfície liquidus assim calculada, que passou a ser empregada em um modelo de controle de processo. A Figura la mostra os resultados da regressão multivariada comparados com os resultados dos cálculos por termodinâmica computacional. A Figura $1 \mathrm{~b}$ apresenta a evolução da temperatura liquidus calculada, ao longo do tempo, após a implantação da formula desenvolvida no controle do processo da aciaria.(6) É evidente que, o uso do modelo de cálculo permitiu controlar as adições de MgO de modo a elevar a temperatura liquidus da escória, garantindo escórias saturadas em MgO nas temperaturas de fim de sopro.

\subsubsection{Controle de inclusões não-metálicas em aços especiais}

Alguns aços modernos (tais como aços para cordoalha de pneus e aços para molas de válvula de motores de combustão interna, por exemplo) têm requisitos de limpeza interna extremamente rigorosos. Com alguma freqüência, os critérios de limpeza exigem a formação de inclusões de alta deformabilidade (baixo ponto de fusão, de forma geral) e a eliminação de inclusões ricas em alumina (importante para a vida à fadiga, por exemplo). $N$ estes casos, adições de alumínio não são desejadas e o controle da absorção de alumínio (proveniente de contaminação da escória ou de ferroligas) é critico para se garantir a formação das inclusões desejadas. Em trabalhos anteriores, alguns exemplos destes controles foram discutidos. (7-9)

Assim, por exemplo, para um aço contendo $0,8 \% C$ $0,6 \% \mathrm{Mn}$ e $0.3 \% \mathrm{Si}$, é interessante garantir que as inclusões formadas no resfriamento, no sistema $\mathrm{MnO}-\mathrm{SiO}_{2}-\mathrm{Al}_{2} \mathrm{O}_{3}$ tenham baixo ponto de fusão (visando deformabilidade na conformação posterior do arame). É possível calcular as concentrações de alumínio (e oxigênio) em solução no aço que conduzirão a estas

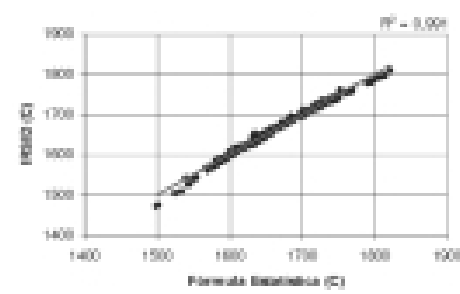

Figura 1a. Correlação entre T liquidus calculada (Thermo-calc, modelo IRSID) e fórmula estatística desenvolvida.

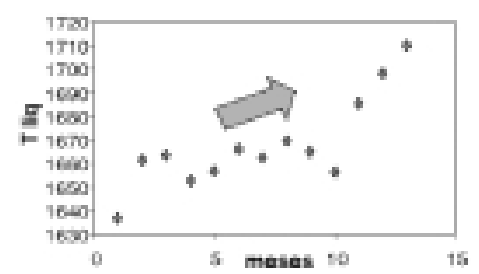

Figura 1b. Evolução da $T$ liquidus da escória no programa de proteção de refratários de uma usina siderúrgica. inclusões, como mostra a Figura 2a. A obtenção destes teores de alumínio (e oxigênio) em solução só é possível através do controle cuidadoso da composição da escória do forno panela. As Figuras $2 b$ e $2 c$ mostram o efeito de duas variáveis importantes da escória de forno panela (basicidade binária e teor de alumina) sobre os teores de alumínio e oxigênio dissolvidos no aço em questão.

Com estas informações é relativamente fácil selecionar composições de escórias de forno panela que resultem nos baixos teores de alumínio em solução no aço necessários a evitar a formação de óxidos de alto ponto de fusão (Figura 2a). Estas composições são 0 ponto de partida para desenvolver, experimentalmente, 0 processo de elaboração.

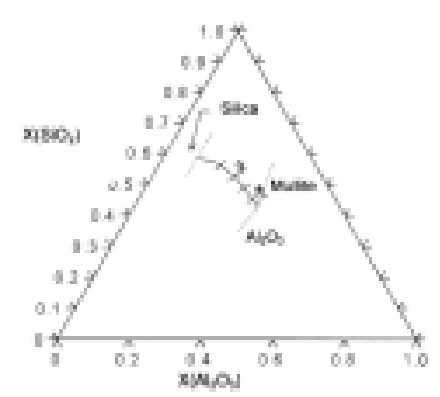

Figura 2a. Equilíbrio aço $(0,8 \% \mathrm{C}, 0,6 \% \mathrm{Mn}$ e $0.3 \% \mathrm{Si})$ $\mathrm{MnO}-\mathrm{SiO}_{2}-\mathrm{Al}_{2} \mathrm{O}_{3}$ a $1823 \mathrm{~K}$. As linhas tracejadas indicam saturação na fase sólida indicada. $\mathrm{N}$ a região entre as linhas tracejadas ocorrem óxidos líquidos. A linha sólida indica 0 teor de Alumínio no aço, em ppm. ${ }^{(8)}$

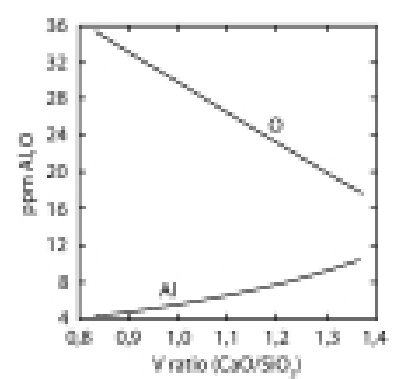

Figura $\mathbf{2 b}$. Efeito da basicidade da escória sobre o teor de Al e $\mathrm{O}$ do aço $\left(\% \mathrm{Al}_{2} \mathrm{O}_{3}=5\right)$

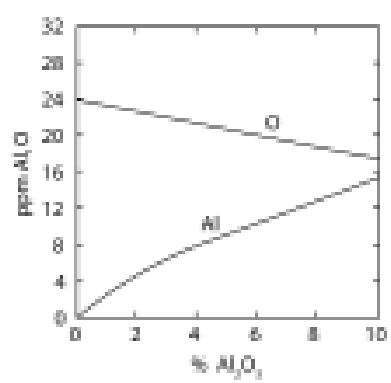

Figura 2c. Efeito do teor de $\mathrm{Al}_{2} \mathrm{O}_{3}$ na escória sobre o teor de $A l$ e 0 do aço $(V=1,2)$. 
Os cálculos de equilíbrio aço-inclusões (Figura 2a) e aço-escória (Figuras 2b e 2c) seriam extremamente difíceis (ou impossíveis) através dos métodos clássicos, em função da dificuldade de prever as atividades dos diversos óxidos (nas inclusões e nas escórias) e da complexidade matemática da expressão das interações dos solutos no ferro líquido. Usando a termodinâmica computacional, o aciarista se concentra, durante a formulação do problema, em decidir quais são as variáveis sobre as quais têm fácil controle (p.ex. os teores de $\mathrm{Si}, \mathrm{Mn}$ e $\mathrm{C}$ do aço e a basicidade da escória, no caso) e quais as variáveis que resultarão do processamento até 0 equilíbrio e que definirão as inclusões formadas.

Também em diversos outros casos, a seleção e o uso de escórias em forno panela tem sido uma ferramenta crítica no desenvolvimento de aços especiais, em que a limpeza interna é critica. Em alguns aços para construção mecânica de elevada limpeza interna, os teores totais de alumínio e oxigênio são críticos para a resistência a fadiga. No desenvolvimento do roteiro de elaboração destes aços, é necessário escolher escórias que resultem em baixos teores destes elementos, no forno panela. A Figura 3 mostra uma comparação entre valores calculados por termodinâmica computacional e medidos no desenvolvimento de um roteiro de elaboração para um aço desta classe. A comparação indica que os resultados dos cálculos se aproximam bastante dos valores medidos. Estes cálculos envolvem o equilíbrio do aço contendo, pelo menos, os solutos Al, O, Ca, Mg, Si, C, Cr e Mn com uma escória contendo, principalmente, $\mathrm{MgO}, \mathrm{CaO}, \mathrm{Al}_{2} \mathrm{O}_{3}$ e $\mathrm{SiO}_{2}$ e $\mathrm{FeO}$.

A realização destes cálculos de equilíbrio aço-escória por métodos clássicos é quase impossível, especialmente em função da dificuldade de prever as atividades dos óxidos na escória.

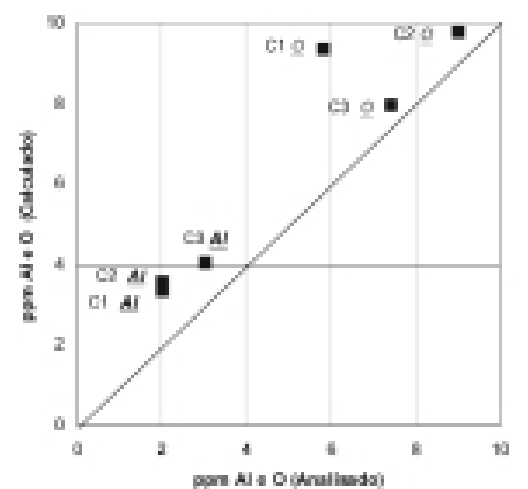

Figura 3. Resultados experimentais ( 3 corridas, $\mathrm{C} 1$ a 3 ) e valores calculados de $\mathrm{Al}$ e $\mathrm{O}$ em solução (1823 K), em equilíbrio com a escória de forno panela, para um aço C-Cr-Mn.

\subsubsection{Segregação na solidificação}

0 conhecimento das condições de redistribuição de soluto durante a solidificação é importante para a definição das condições de lingotamento contínuo, parâmetros de tratamento termomecânico posterior e garantia da homogeneidade desejada no produto. É bem estabelecido que a solidificação dos aços, em escala industrial, não ocorre em equilíbrio. Entretanto, em casos especiais, o modelo de Scheil (que assume homogeneização completa no liquido e nenhuma difusão no sólido) pode fornecer uma boa aproximação da solidificação de aços. Esta aproximação é ainda melhor se for possível considerar que os solutos intersticiais se homogeneizam completamente no sólido, por difusão, no chamado modelo de "equilíbrio parcial". Tanto o modelo de Scheil como o modelo "equilíbrio parcial" estão implementados em programas de termodinâmica computacional como Thermo-calc.(10)

A Figura 4a, de Chen e Sundman(10) compara diferentes modelos aplicados à redistribuição de solutos durante a solidificação do aço M2. Tanto o modelo de Scheil (indicado como Scheil-Gulliver) como o modelo de equilíbrio parcial (indicado como Partial Equilibrium) prevêem corretamente a seqüência de fases formadas. Adicionalmente, 0 modelo de equilíbrio parcial prevê, com razoável precisão, a temperatura solidus da liga.

A Figura $4 b$ apresenta o perfil de composição química calculado para o $\mathrm{Mn}$ em um aço $\mathrm{C}-\mathrm{Mn}$ solidificado a $2.7 \mathrm{~K} / \mathrm{min}$, empregando um programa que acopla termodinâmica computacional a cálculos de difusão (DICTRA). Os valores calculados aproximam-se bastante dos valores experimentais medidos por Mizoguchi(11) nas mesmas condições. Quando se assume que a razão de partição dos solutos é conhecida e constante, o modelo de Scheil pode ser implementado com relativa facilidade em programas simples de computador. A consideração da partição real depende, entretanto, da aplicação da termodinâmica computacional a cada temperatura considerada.

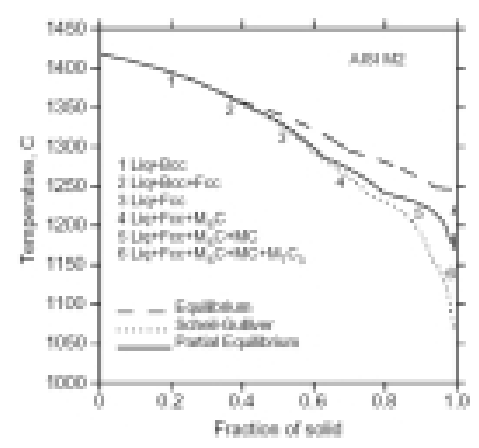

Figura 4a. Modelamento da solidificação do aço M2 por diferentes técnicas. $\grave{A}$ direita, medidas de T solidus $\boldsymbol{\Lambda}$.(10)

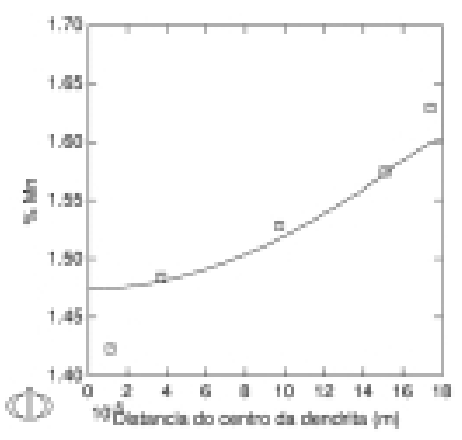

Figura 4b. Perfil calculado de $\mathrm{Mn}$ em aço com $\% \mathrm{C}=0.13 \% \mathrm{Si}=0.35 \% \mathrm{Mn}=1.52$, considerando a difusão no sólido (DICTRA). Dados de Mizoguchi. (11) 


\section{CONCLUSÕES}

A aplicação de métodos computacionais a solução de problemas de termodinâmica evoluiu consideravelmente nas ultimas décadas do século passado. Hoje, os programas e bancos de dados disponíveis são ferramentas extremamente úteis para a solução de problemas reais de aciaria. À medida que os requisitos sobre a qualidade dos aços se tornam mais rigorosos e os custos de experiências se elevam, esta ferramenta encontra cada vez mais aplicação para a previsão de condições recomendáveis para a elaboração e processamento do aço. É possível supor que o ensino de termodinâmica para siderurgistas precise passar por uma significante revisão, de modo a enfatizar os conceitos necessários à aplicação segura destes métodos, reduzindo o ensino de métodos excessivamente simplificados de solução matemática de problemas.

O s métodos desenvolvidos para a solução dos problemas de termodinâmica computacional estão sendo estendidos para incluir o tratamento de problemas de difusão, com resultados extremamente satisfatórios em varias áreas, incluindo, especialmente, o modelamento da redistribuição de soluto na solidificação.

\section{Lista de Símbolos}

$\Delta G_{i}{ }^{1 \%}$ Energia livre de solução no ferro líquido para $1 \%$ em massa de i (12) (variação de energia livre molar na formação de uma solução diluída, hipotética, a $1 \%$ em massa do soluto i)

$\gamma_{i}^{0} \quad$ Coeficiente de atividade do soluto i no ferro líquido, na Lei de Henry (12) (valor do coeficiente de atividade do soluto i à diluição infinita)

$a_{i} \quad$ Atividade do soluto i (atividade henriana do soluto i baseada em um estado de referência hipotético de $1 \%$ em massa de i em solução no ferro líquido) (12)

$M_{i} \quad$ Massa atômica do elemento i

$R \quad$ Constante dos gases

$T$ Temperatura, em K

$f_{i} \quad$ Coeficiente de atividade (henriana) do soluto i (para concentração em \% em massa)

$\%_{i} \quad$ Percentagem em massa do soluto i no ferro líquido

$e_{i}^{j} \quad$ Coeficiente de interação entre os solutos i e j (primeira ordem) (concentração em \% em massa)

$r_{i}^{j} \quad$ Coeficiente de interação entre os solutos i e j (segunda ordem) (concentração em \% em massa)

$P \quad$ N úmero de fases em um sistema

$F \quad$ Número de graus de liberdade em um sistema

$C \quad$ Número de constituintes em um sistema

$V \quad$ Basicidade binária de escória

\section{REFERÊNCIAS}

1 WAGNER, C. Thermodynamics of alloys. Reading, MA : Addison-Wesley, 1952.

2 CHIPMAN, J.; ELLIOT, J.F. The thermodynamics of liquid metallic solutions. In: physical metallurgy. Cleveland : ASM, 1950.

3 KAPOOR, M.L.; FROHBERG, M.G. Theoretical treatment of activities in silicate melts. In: CHEMICAL MetalluRgy OF IRON AND STEel CONFEREN CE, 1971. Proceedings... London : University of Sheffield, 1971.

4 HEALY, G.W. A new look at phosphorus distribution. Journal of the Iron and Steel Institute, v. 208, n. 6, p. 664668, June 1970.

5 COSTA E SILVA, A. Representação de dados termodinâmicos de soluções em siderurgia, In: CO N GRESSO AN U AL DA ABM, 51., 1996, Porto Alegre. Anais... São Paulo : ABM, 1996.

6 CHRISÓ STO MO, W.B.; PEREIRA, C.L.; COSTA E SILVA, A. Avaliação da solubilidade de MgO em escórias de aciaria através de diferentes métodos de cálculo. In: SEMIN ÁRIO DE FUSÃO, REFIN O E SO LIDIFICAÇÃO DOS METAIS, 30., 1999, Belo Horizonte. Anais... São Paulo: ABM, 1999.

7 PIMEN TEL, M.F.; COSTA E SILVA, A.; PRADO, E.L.; SALO MÃO JR., W. Efeitos das práticas de elaboração do aço sobre a limpeza interna dos aços para mola de válvula (SAE6150 e SAE 9254). In: CON GRESSO AN UAL DA ABM, 54., 1999, São Paulo. Anais... São Paulo : ABM, 1999.

8 O ERTEL, L.; COSTA E SILVA, A. Application of thermodynamic modeling to slag-metal equilibria in steelmaking. CALPHAD, v. 23, n.3-4, 1999. 
9 COSTA E SILVA, A. An overview of the use of CALPHAD methods in steelmaking. Journal of Mining and Metallurgy, v. 35B, n.1, p.85-112, 1999.

10 CHEN, Q.; SUN DMAN ,B. Computation of partial equilibrium solidification with complete interstitial and negligible substitutional back diffusion. Materials Transactions Japan Institute for Metals, v. 43, n.3, p. 551-559, 2002.

11 MIZOGUCHI, S. A study on segregation and oxide inclusions for the control of steel properties. Tokyo : University of Tokyo, 1996. p. 97.

12 TURKD O GAN, E.T. Fundamentals of steelmaking, London : The Institute of Materials, 1996.

Recebido em: 12/12/2006

Aceito em: 27/10/2006

Proveniente de: SEMIN ÁRIO DE FUSÃO, REFIN O E SO LIDIFICAÇÃO DOS METAIS - IN TERN ACIO N AL, 36., 2005, Vitória, ES. São Paulo : ABM, 2005. 